University of Victoria

ISSN 1485-6441

Department of Economics

\title{
Nonjointness and Scope Economies in the Multiproduct Symmetric Generalized McFadden Cost Function
}

\author{
Kenneth G. Stewart \\ Department of Economics, University of Victoria \\ Victoria, B.C., Canada V8W 2 Y2
}

November 2007

\begin{abstract}
The multiproduct symmetric generalized McFadden cost function is increasingly prominent in empirical production analysis. Researchers should be aware that the scope for imposing and testing nonjointness in this model is limited. In the general version of the model, nonjointness requires the nontestable maintained hypothesis of similar (in a sense we define) single-output production technologies, a maintained hypothesis for which there is not normally any basis. The apparent imposition and testing of nonjointness must be qualified accordingly. In restricted versions of the model involving separability or constant returns that are often of interest in applied work, nonjointness is precluded.
\end{abstract}

Keywords: $\quad$ Nonjointness, multiproduct cost function

JEL Classifications: C50, D24

\section{Author Contact:}

Kenneth G. Stewart, Dept. of Economics, University of Victoria, P.O. Box 1700, STN CSC, Victoria, B.C., Canada V8W 2Y2; e-mail: kstewart@uvic.ca; Voice: (250) 721-8534; FAX: (250) 721-6214 
Estimated demand parameters often serve as inputs to policy analysis, such as the computational general equilibrium modeling of tax changes. For such purposes, it is important that the parameter estimates satisfy the properties dictated by economic theory, such as demand homogeneity, symmetry conditions arising from the assumed twice-differentiability of the objective function, and curvature conditions.

Because unrestricted demand estimates often violate at least some of these properties, they typically need to be imposed in estimation. Whereas this is generally straightforward in the case of homogeneity and symmetry, it is more problematic in the case of curvature conditions. Diewert and Wales $(1987,1988)$ and Diewert and Ostensoe (1988) proposed flexible functional forms for, respectively, cost, expenditure, and profit functions, in which the associated demand system can be restricted to satisfy concavity without destroying the flexibility of the functional form. In applications requiring the imposition of concavity, it seems fair to say that the Diewert-Wales functional forms are now the received class of econometric demand models. ${ }^{1}$

In the case of production analysis, an issue arises that has no parallel in consumer demand: production often involves more than one output. The Diewert-Wales (1987) symmetric generalized McFadden (SGM) cost function was extended to the multiproduct case by Kumbhakar (1994). Prior to Kumbhakar's contribution, the dominant multiproduct factor demand model was the translog. However, in addition to being able to impose concavity without destroying flexibility, Kumbhakar's multiproduct SGM (MSGM) model inherits from the Diewert-Wales single-output model at least two other advantages over the translog.

First, all the parameters of the MSGM cost function appear in the associated system of factor demands. Thus the technology can be fully recovered from the estimation of the factor demands alone. This contrasts with the translog model, where key parameters of the cost function relating to the role of output are lost from the factor demands. Consequently some important hypotheses and elasticities - such as those relating to returns to scale - require the estimation of the translog cost function joint with its system of factor demands.

A second advantage of the MSGM model is that in a translog factor demand system prices and outputs appear in logarithmic form, and so zero values are not permitted; this is not the case for MSGM demands. Particularly in multiproduct applications, it is not unusual for some outputs to take on zero values for some observations in the sample, lending a key 
advantage to the MSGM model.

For all these reasons, to at least the extent that the original Diewert-Wales SGM model has become prominent in single-output production analysis, Kumbhakar's MSGM cost function has come to dominate recent work on multi-output production; examples include Asai (2006), Døhl (2002), Larsson (2003), Peeters and Surry (2000), and Stewart and Jones (2005).

However any functional form - even ones that are flexible in the sense of Diewert (1974), as the SGM and MSGM models are-has limitations in the features of technology that can be imposed and tested. The purpose of any empirical study of multi-output production is, at least in part, to understand the nature of the transformation of multiple inputs into multiple outputs; the general principles governing such transformations are exposited in the classic article by Hall (1973). The present paper investigates the testable implications of the MSGM model as they relate to joint production by interpreting the model in terms of Hall's analysis. We find that, whereas the MSGM model is admirable in its ability to test output separability, its ability to to test input nonjointness is more limited.

\section{The Single- and Multi-Output SGM Cost Functions}

Let us begin by establishing notation, for the most part adopting the original conventions of Diewert and Wales (1987) and Kumbhakar (1994) and modifying Hall's (1973) notation accordingly. Many applications of these models study technical change, and the issues surrounding this appear to be well understood in the literature. Our focus is instead on the economics of joint production, and so we drop the technical change terms from the cost functions as they appear in the original sources rather than carry them through the analysis.

Consider a production technology denoted in general by $t(\boldsymbol{y}, \boldsymbol{x})$ that transforms inputs $\boldsymbol{x} \equiv\left[x_{1} ; x_{2} ; \cdots ; x_{n}\right]$ into outputs $\boldsymbol{y} \equiv\left[y_{1} ; y_{2} ; \cdots ; y_{m}\right]$; following the production literature, we denote the input prices by $\boldsymbol{p} \equiv\left[p_{1} ; p_{2} \cdots ; p_{n}\right]$.

Beginning with the special case in which $\boldsymbol{y}$ consists of just a single output $y$, the SGM cost function of Diewert and Wales (1987) is ${ }^{2}$

$$
c(y, \boldsymbol{p})=g(\boldsymbol{p}) \beta y+\sum_{i} b_{i} p_{i}+\beta y \sum_{i} b_{i i} p_{i}+d\left(\sum_{i} \lambda_{i} p_{i}\right) y^{2},
$$

where

$$
g(\boldsymbol{p}) \equiv \frac{\boldsymbol{p}^{\prime} \boldsymbol{S} \boldsymbol{p}}{2 \boldsymbol{\theta}^{\prime} \boldsymbol{p}}
$$

For reasons that will become apparent, we have supplemented the Diewert-Wales specification by introducing the parameter $\beta$, although it is not identified in this single-output model; Diewert and Wales effectively normalize it to 1 . Beyond this, to the extent that notation is not self-evident the reader is referred to the original source for definitions. Absent 
other restrictions yielding appropriate special cases, such as homotheticity, the parameters of the last term require an additional restriction to be identified; because the $\lambda_{i}$ end up in the conditional factor demands, Diewert and Wales (pp. 58-59) let this restriction be $d=1$.

A special case that will be of interest is that of a homothetic technology, where the single-output cost function has the multiplicatively separable form

$$
c(y, \boldsymbol{p})=h(y) \phi(\boldsymbol{p}) .
$$

It is evident that homotheticity in the SGM cost function requires the restrictions $d=b_{i}=0$ $(i=1, \ldots, n)$, which reduce the function to

$$
c(y, \boldsymbol{p})=\left(g(\boldsymbol{p}) \sum_{i} b_{i i} p_{i}\right) \beta y .
$$

This is homogeneous of degree 1 in output, and so exhibits constant returns to scale (CRS). Thus in the single-output SGM model, although not for cost functions generally, the homotheticity and CRS restrictions are one and the same.

Turning to the multiproduct generalization formulated by Kumbhakar (1994), the MSGM cost function is

(4) $C(\boldsymbol{y}, \boldsymbol{p})=g(\boldsymbol{p}) \sum_{k} \beta_{k} y_{k}+\sum_{i} b_{i} p_{i}+\left(\sum_{k} \beta_{k} y_{k}\right) \sum_{i} b_{i i} p_{i}+\left(\sum_{i} \lambda_{i} p_{i}\right) \sum_{j} \sum_{k} d_{j k} y_{j} y_{k}$.

The $d_{j k}$ are required to be symmetric: $d_{j k}=d_{k j}$. In analogy with the single-output case, in the absence of other restrictions yielding appropriate special cases of the kinds considered below, identification requires a linear restriction on the $\beta_{k}$ and on the $d_{j k}$.

Also in analogy with the single-output case, CRS is imposed on the MSGM model with the restrictions $d_{j k}=b_{i}=0$ for all $i, j, k$. This reduces the cost function to

$$
C(\boldsymbol{y}, \boldsymbol{p})=\left(g(\boldsymbol{p})+\sum_{i} b_{i i} p_{i}\right)\left(\sum_{k} \beta_{k} y_{k}\right),
$$

so that it is homogeneous of degree 1 in the outputs.

In what sense is the SGM model (1) a special case of the MSGM model? Consider a multiproduct firm that produces amount $y_{k}$ of output $k$ but nothing of all its other products. According to the MSGM cost function, its costs are

$$
\begin{aligned}
c^{(k)}\left(y_{k}, \boldsymbol{p}\right) & \equiv C\left(0, \ldots, 0, y_{k}, 0, \ldots, 0 ; \boldsymbol{p}\right) \\
& =g(\boldsymbol{p}) \beta_{k} y_{k}+\sum_{i} b_{i} p_{i}+\beta_{k} y_{k} \sum_{i} b_{i i} p_{i}+\left(\sum_{i} \lambda_{i} p_{i}\right) d_{k k} y_{k}^{2} .
\end{aligned}
$$

This is the single-output function (1) applied to the $k$ th output.

With these preliminaries as background, we now turn to the restrictions particular to joint production: output separability and input nonjointness. ${ }^{3}$ Interpretation in terms of 
Hall's analysis is facilitated by stating these restrictions in terms of the cost function. We leave it understood that in practice they would be implemented by imposing them in estimation on the factor demands obtained via Shephard's lemma.

\section{Output Separability}

It is helpful to begin with the case in which the issues are straightforward. A technology $t(\boldsymbol{y}, \boldsymbol{x})$ is said to be output separable if it decomposes additively as $t(\boldsymbol{y}, \boldsymbol{x})=-h(\boldsymbol{y})+f(\boldsymbol{x})$. In these circumstances Hall's (1973, p. 890) General Theorem on Separability applies: the joint cost function has the form

$$
C(\boldsymbol{y}, \boldsymbol{p})=C^{*}(h(\boldsymbol{y}), \boldsymbol{p}) .
$$

Effectively, then, separability is the notion that although the firm produces several outputs, this can be modeled as the production of a single aggregate output $h(\boldsymbol{y})$.

... with a Linear Aggregator Separability can be imposed on the MSGM model by the restrictions $d_{j k}=\beta_{j} \beta_{k}$ (Kumbhakar equ. (5)). Under these restrictions the double summation in the final term of (4) simplifies to

$$
\sum_{j} \sum_{k} \beta_{j} \beta_{k} y_{j} y_{k}=\left(\sum_{j} \beta_{j} y_{j}\right)\left(\sum_{k} \beta_{k} y_{k}\right)=\left(\sum_{k} \beta_{k} y_{k}\right)^{2} .
$$

Thus the entire cost function can be expressed in terms of the linear production aggregate $h(\boldsymbol{y})=\sum_{k} \beta_{k} y_{k}$. This aggregate then plays the same role in the MSGM model as does $y$ in the single-output cost function (1) (in which the identifying normalizations $\beta=d=1$ are imposed), and Hall's General Theorem on Separability is manifested.

Although the restrictions $d_{j k}=\beta_{j} \beta_{k}$ are the weakest ones yielding separability, the function can be separable in other circumstances as well. Specifically, the CRSconstrained function (5) is also separable in the linear aggregator. Thus separability does not imply CRS but, in the MSGM cost function (although not for cost functions generally), CRS implies separability.

$\ldots$ with an Additive Aggregator Consider the even stronger restrictions $\beta_{k}=1$ and $d_{j k}=d$ (Kumbhakar, equ. (4)). Imposing these on the MSGM model, the final term of the cost function simplifies to

$$
\sum_{j} \sum_{k} d y_{j} y_{k}=d\left(\sum_{j} y_{j}\right)\left(\sum_{k} y_{k}\right)=d\left(\sum_{k} y_{k}\right)^{2} .
$$

Thus the entire cost function can be expressed in terms of the additive aggregate $h(\boldsymbol{y})=\sum_{k} y_{k}$. Once again, this aggregate then plays the same role as does $y$ in the single-output function. 
Because $d$ must be normalized to a known arbitrary (nonzero) constant for the $\lambda_{i}$ to be identified, this set of restrictions can be stated equivalently as $\beta_{k}=1, d_{j k}=\beta_{j} \beta_{k}=1$, which makes it clear that this is a more restrictive condition than, and is nested within, output separability with a linear aggregator.

Economically, the hypothesis of an additive aggregator is a considerably more restrictive one than the hypothesis of a linear aggregator. Whereas a linear aggregator might be a reasonable approximation for many multiproduct technologies, an additive aggregator is much less likely to be appropriate, especially when outputs are measured in real rather than value units. We would therefore expect additive aggregation to be rejected in many applied contexts, whereas linear aggregation might not be.

\section{$3 \quad$ Input Nonjointness}

Input nonjointness is the notion that there are no cost economies from combining the production of the outputs: the costs of multi-output production are the same as the costs of producing the outputs individually. It has the implication that the multiproduct cost function can, without loss of generality, be modeled by its single-output counterparts. Note that this is a distinct concept from output separability. To say that total output can be treated as a linear or additive aggregate of individual outputs is a different matter from saying that there are no cost economies in the joint production of the goods.

Let the single-output cost function for $y_{k}$ be denoted $c^{(k)}\left(y_{k}, \boldsymbol{p}\right), k=1, \ldots, m$, as in (6). The formal definition of input nonjointness stated in terms of the cost function is given by Hall's (1973, p. 890) General Theorem 2 on Nonjointness: a technology is nonjoint if and only if the joint cost function can be written as the sum of independent cost functions for each kind of output:

$$
C(\boldsymbol{y}, \boldsymbol{p})=\sum_{k=1}^{m} c^{(k)}\left(y_{k}, \boldsymbol{p}\right) .
$$

Chambers (1988, p. 293) gives as a requirement for nonjointness that

$$
\frac{\partial^{2} C}{\partial y_{j} \partial y_{k}}=0 \quad \text { for all } j, k, j \neq k \text {. }
$$

For the MSGM model this yields the restrictions $d_{j k}=0(j \neq k)$, under which the double summation in the final term of (4) simplifies so as to reduce the function to

$$
C(\boldsymbol{y}, \boldsymbol{p})=g(\boldsymbol{p}) \sum_{k} \beta_{k} y_{k}+\sum_{i} b_{i} p_{i}+\left(\sum_{k} \beta_{k} y_{k}\right) \sum_{i} b_{i i} p_{i}+\left(\sum_{i} \lambda_{i} p_{i}\right) \sum_{k} d_{k k} y_{k}^{2} .
$$

Now suppose that all outputs are produced by single-output technologies having cost functions of the Diewert-Wales form (1). We assume further these SGM cost functions have 
common values for the parameters $b_{i i}, \lambda_{i}$, and those that appear in $g(\boldsymbol{p})$; the remaining parameters $b_{i}, \beta$, and $d$ are permitted to be product-specific and are denoted $b_{i}^{(k)}$ and (as in (6)) $\beta_{k}, d_{k k}$. The total of single-output costs is

$$
\begin{aligned}
\sum_{k} c^{(k)}\left(y_{k}, \boldsymbol{p}\right)= & g(\boldsymbol{p}) \sum_{k} \beta_{k} y_{k}+\sum_{i}\left(\sum_{k} b_{i}^{(k)}\right) p_{i} \\
& +\left(\sum_{k} \beta_{k} y_{k}\right) \sum_{i} b_{i i} p_{i}+\left(\sum_{i} \lambda_{i} p_{i}\right) \sum_{k} d_{k k} y_{k}^{2}
\end{aligned}
$$

Comparing this with (10) reveals that, with the interpretation of the coefficients $b_{i}$ of the MSGM model as being the aggregates $b_{i} \equiv \sum_{k} b_{i}^{(k)}$, Hall's definition of nonjointness is satisfied. Equally evident, however, is that, whereas this aggregation works for product-specific values of the $b_{i}^{(k)}$, it would not similarly work for product-specific values of the $b_{i i}, \lambda_{i}, \boldsymbol{S}$, or $\boldsymbol{\theta}$ parameters because of the manner in which they enter. ${ }^{4}$ Nonjointness therefore requires not just that the restrictions $d_{j k}=0(j \neq k)$ hold, but also that the production technologies be similar in the sense that they share common values of these parameters. Because potentially product-specific values of these parameters are underidentified, there are implications for our ability to study nonjointness.

Only in a qualified sense can nonjointness be imposed or tested. Estimation of the MSGM model subject to the restrictions $d_{j k}=0(j \neq k)$ imposes nonjointness in the only sense in which it is possible to do so, but with the understanding that the similar production technologies that are implied by the claim that nonjointness has been imposed are by assumption. In the same way, to say that a test of the restrictions constitutes a test of nonjointness must not be understood to mean that the similarity of technologies has been tested; instead the similarity of technologies (as we have defined it) is a maintained hypothesis on which this test of nonjointness is conditional. A rejection of the restrictions $d_{j k}=0(j \neq k)$ is properly interpreted as a rejection of nonjointness because, even if the maintained hypothesis is true, nonjointness requires the restrictions as well. But to interpret a failure to reject the restrictions as support for nonjointness involves the implicit maintained hypothesis of similar technologies, a maintained hypothesis for which there is not normally any basis.

An interesting contrast in which these subtleties of interpretation disappear is the generalized linear-generalized Leontief cost function that Hall (1973) uses for illustrative purposes. $^{5}$ In that model all parameters are permitted to be output-specific and so the nonjointness restrictions include ones for identical technologies. 


\subsection{Economies of Scope}

Another way of interpreting this conclusion is in terms of economies of scope. The standard measure is (Baumol, Panzar, and Willig, 1982)

$$
\mathrm{ESCP}=\frac{\sum_{k} C\left(0, \ldots, 0, y_{k}, 0, \ldots, 0 ; \boldsymbol{p}\right)}{C(\boldsymbol{y}, \boldsymbol{p})} .
$$

For the MSGM cost function, the terms in the numerator are given by (6), the denominator by (4):

$$
\mathrm{ESCP}=\frac{g(\boldsymbol{p}) \sum_{k} \beta_{k} y_{k}+m \sum_{i} b_{i} p_{i}+\left(\sum_{k} \beta_{k} y_{k}\right) \sum_{i} b_{i i} p_{i}+\left(\sum_{i} \lambda_{i} p_{i}\right) \sum_{k} d_{k k} y_{k}^{2}}{g(\boldsymbol{p}) \sum_{k} \beta_{k} y_{k}+\sum_{i} b_{i} p_{i}+\left(\sum_{k} \beta_{k} y_{k}\right) \sum_{i} b_{i i} p_{i}+\left(\sum_{i} \lambda_{i} p_{i}\right) \sum_{j} \sum_{k} d_{j k} y_{j} y_{k}} .
$$

Although this is certainly a legitimate measure of economies of scope in the MSGM context, as reflected by its application in several of the empirical papers cited in the introduction, note that it does not reduce to 1 under the restrictions $d_{j k}=0(j \neq k)$. Although the denominator reduces to (10), it still differs from the numerator owing to the factor $m$ in the $\sum_{i} b_{i} p_{i}$ term. Thus estimating the MSGM cost function subject to the restrictions $d_{j k}=0$ $(j \neq k)$ does not impose a unitary ESCP.

A quite different approach to testing nonjointness might appear to be offered by the fact that ESCP is a computable statistic, and so a standard error can be obtained for it as in, for example, Asai (2006). Given that confidence bounds can therefore be computed for ESCP, why not interpret bounds that do not include $\mathrm{ESCP}=1$ as a rejection of nonjointness? The problem with this is that, although nonjointness is one source of economies of scope, it is not the only possible source; see Baumol, Panzar, and Willig (1982, pp. 75-79). Thus, although the MSGM model permits economies (or diseconomies) of scope, it does not ascribe those economies to particular sources, nonjointness or otherwise, at least in a way that is testable.

\section{Nonjointness, Separability, and CRS}

How are these conclusions affected by special cases of the MSGM cost function, such as versions involving separability or constant returns? The answer turns out to depend less on issues surrounding non-identical single-output technologies than on another of Hall's results, an impossibility theorem on separable nonjoint technologies which states that a CRS technology cannot be both separable and nonjoint.

Prior to invoking this impossibility theorem, let us begin with Hall's (p. 891) General (in the sense that it applies in the absence of CRS) Theorem on Separable Nonjoint Technologies: a technology is both separable and nonjoint if and only if the cost function has the structure

$$
C(\boldsymbol{y}, \boldsymbol{p})=\phi(\boldsymbol{p}) \sum_{k=1}^{m} h^{(k)}\left(y_{k}\right)
$$


That is, the separable cost function (7) must factor as

$$
C^{*}(h(\boldsymbol{y}), \boldsymbol{p})=h(\boldsymbol{y}) \phi(\boldsymbol{p}) \quad \text { where } h(\boldsymbol{y})=\sum_{k=1}^{m} h^{(k)}\left(y_{k}\right) .
$$

Equivalently, the product-specific cost functions in the nonjoint aggregation (9) must have the homothetic form (2) in which the $\phi(\boldsymbol{p})$ component is common: $c^{(k)}\left(y_{k}, \boldsymbol{p}\right)=h^{(k)}\left(y_{k}\right) \phi(\boldsymbol{p})$. The implication is that the separate production functions to which the $c^{(k)}\left(y_{k}, \boldsymbol{p}\right)$ are dual are identical except for pure scale effects.

\section{Nonjointness and Separability}

Now consider the MSGM cost function (4). We have seen that imposing CRS on it yields the separable form (5), but that the model can be separable (as in (8)) without requiring CRS. But although separability does not imply CRS, it turns out that attempting to impose on the separable model the restrictions that appear to be associated with nonjointness has the effect of imposing CRS; by Hall's impossibility theorem, nonjointness is precluded.

To see this, consider the MSGM cost function in its separable form (8) based on a linear aggregator. What further restrictions on the MSGM function are needed to yield nonjointness? Hall's theorem tells us that the output-specific cost functions must be homothetic, and therefore of the form (3):

$$
c^{(k)}\left(y_{k}, \boldsymbol{p}\right)=h^{(k)}\left(y_{k}\right) \phi(\boldsymbol{p})=\left(g(\boldsymbol{p})+\sum_{i} b_{i i} p_{i}\right) \beta_{k} y_{k} .
$$

Note that we have tentatively permitted $\phi(\boldsymbol{p})=g(\boldsymbol{p})+\sum_{i} b_{i i} p_{i}$ to be common across the outputs, so that, for the moment, product-specific parameter values in $\phi(\boldsymbol{p})$ are not an impediment to aggregation.

Under the restrictions $\lambda_{i}=b_{i}=0$, the separable MSGM cost function yielded by ( 8 ) reduces to the sum of the homothetic functions,

$$
C(\boldsymbol{y}, \boldsymbol{p})=\left(g(\boldsymbol{p})+\sum_{i} b_{i i} p_{i}\right)\left(\sum_{k} \beta_{k} y_{k}\right)=\phi(\boldsymbol{p}) \sum_{k} h^{(k)}\left(y_{k}\right),
$$

and so Hall's definition (11) is satisfied. These would therefore appear to be the restrictions for nonjointness conditional on separability. But note that this version of the cost function is the CRS-constrained form (5); a test of the restrictions $\lambda_{i}=b_{i}=0$ in the separable model (8) is therefore properly interpreted as a test of CRS conditional on separability. By Hall's impossibility theorem, given that the model is both separable and has constant returns, it cannot also be nonjoint.

The paradox of this conclusion is striking. Even if we assume identical output-specific production technologies as defined in Hall's General Theorem on Separable Nonjoint Technologies, the parameter restrictions on the separable MSGM cost function that would appear 
to be associated with nonjointness not only do not provide a test of that hypothesis, they actually preclude nonjointness from holding.

\section{Nonjointness and CRS}

Finally, let us note for completeness that these conclusions are unchanged even if we set aside separability and consider only the special case of constant returns. As we noted in (5), CRS imposed on the MSGM cost function implies separability, and so by Hall's impossibility theorem nonjointness cannot also hold. Thus in the MSGM cost function with CRS, not only can nonjointness not be imposed or tested, it is actually precluded from holding.

\section{Conclusions}

Kumbhakar's (1994) multiproduct symmetric generalized McFadden cost function increasingly dominates applied multi-output production analysis, particularly in contexts in which concavity must be imposed or some products take on zero values for some observations. It is therefore useful to be clear about exactly what features of joint production the model allows to be imposed and tested. We have found that, whereas the model is admirable in its ability to test output separability, the ability to test nonjointness involves many qualifications and nuances of interpretation.

This is no criticism of the model. Any parametric representation of economic behavior inevitably involves some limitations, and flexible functional forms are no exception. For example, although we have described the MSGM cost function as admirable in its ability to test separability, this separability must nevertheless be in the form of a linear (or, as a special case, additive) production aggregate; nonlinear aggregates are not permitted. Although this can hardly be viewed as a serious deficiency of the model for most purposes, it is conceivable that for some issues in production analysis it might be.

The point is that, when empirical researchers choose a parametric representation of behavior, they should do so with an understanding of the limitations of their choice. In the case of the MSGM cost function, we have shown that input nonjointness can only be imposed and tested in rather special and limited senses, and the apparent imposition of nonjointness on the model does not imply a unitary measure of economies of scope, at least according to the standard measure. Plausible special cases of the model that are often of interest in practice - involving separability or constant returns - not only do not permit the imposition or testing of nonjointness, they actually preclude it from holding.

As limitations of flexible functional forms go, however, these are fairly benign ones. The MSGM cost function permits economies (or diseconomies) of scope; that it does not attribute these to particular sources - nonjointness or otherwise - in ways that are amenable to formal 
testing is, for most work in applied production analysis, not of great consequence. Similarly, although ideally it would be of interest to be able to impose and test nonjointness unhindered by the limitations we have identified, the reality is that it can rarely be expected to hold in practice. Firms produce multiple outputs precisely because there are cost economies to doing so, and develop over time in part by expanding their product lines in ways that exploit such opportunities. A limited ability to impose and test the absence of such economies is, therefore, a rather modest deficiency of a flexible functional form cost function. 


\section{Notes}

${ }^{1}$ The Diewert-Wales models are not unique in permitting the imposition of concavity, but they have the important feature that they are designed to do so without destroying the flexibility of the functional form. For example, the asymptotically ideal production model of Barnett, Geweke, and Wolfe (1991) also permits concavity to be imposed on the cost function, but Terrell (1995) has shown that this dramatically impairs the flexibility of the form.

${ }^{2}$ To facilitate comparison with the multiproduct model the symbol $d$ is used for the Diewert-Wales parameter $b_{y y}$.

${ }^{3}$ There are parallel notions of output nonjointness and input separability, but these can only be studied in the framework of revenue and profit functions, not cost functions. In this paper we therefore often follow Hall (1973) in using the terms separability and nonjointness to refer to output separability and input nonjointness.

${ }^{4}$ In practice the $\theta_{i}$ are set to the sample means of the factor inputs rather than being estimated. Nevertheless their aggregation remains problematic.

${ }^{5}$ Hall's cost function is primarily for illustrative value because, with the parameters permitted to be output-specific, it involves a very large number of parameters - far more than are needed for flexibility. Although his function is flexible, it is not parsimonious. 


\section{References}

Asai, S. (2006) "Scale economies and scope economies in the Japanese broadcasting market," Information Economics and Policy 18, 321-331.

Barnett, W.A., Geweke, J., and M. Wolfe (1991) "Seminonparametric Bayesian estimation of the asymptotically ideal production model," Journal of Econometrics 49, 5-50.

Baumol, W.J., Panzar, J., and R. Willig (1982) Contestable Markets and the Theory of Industry Structure. San Diego: Harcourt Brace Jovanovich.

Chambers, R.G. (1988) Applied Production Analysis-A Dual Approach. New York: Cambridge University Press.

Diewert, W.E. (1974) "Application of duality theory," in Intriligator, M.D., and D.A. Kendrick (eds.) Frontiers of Quantitative Economics, Vol. 2. Amsterdam: NorthHolland.

Diewert, W.E., and L. Ostensoe (1988) "Flexible functional forms for profit functions and global curvature conditions," in Barnett, W.A., Berndt, E.R., and H. White (eds.) Dynamic Econometric Modeling. New York: Cambridge University Press.

Diewert, W.E., and T.J. Wales (1987) "Flexible functional forms and global curvature conditions," Econometrica 55, 43-68.

Diewert, W.E., and T.J. Wales (1988) "Normalized quadratic systems of consumer demand functions," Journal of Business and Economic Statistics 6, 303-312.

Døhl, Ø, (2002) "Energy flexibility and technological progress with multioutput production," Discussion Paper No. 318, Research Department, Statistics Norway.

Hall, R.E. (1973) "The specification of technology with several kinds of outputs," Journal of Political Economy 81, 878-892.

Kumbhakar, S.C. (1994) "A multiproduct symmetric generalized McFadden cost function," Journal of Productivity Analysis 5, 349-357.

Larsson, J. (2003) "Testing the multiproduct hypothesis on Norwegian aluminum plants," Discussion Paper No. 350, Research Department, Statistics Norway.

Peeters, L., and Y. Surry (2000) "Incorporating price-induced innovation in a symmetric generalized McFadden cost function with several outputs," Journal of Productivity Analysis 14, 53-70. 
Stewart, K.G., and J.C.H. Jones (2005) "Are sports teams multi-product firms?" Econometrics Working Paper EWP0513, Department of Economics, University of Victoria.

Terrell, D. (1995) "Flexibility and regularity properties of the asymptotically ideal production model," Econometric Reviews 14, 1-17. 